\title{
Closed chest modification of atrioventricular conduction system in man for treatment of refractory supraventricular tachycardia
}

\author{
GIUSEPPE CRITELLI, FRANCESCO PERTICONE, FERNANDO COLTORTI, \\ VITTORIO MONDA, JOHN GALLAGHER
}

From the Istituto di Patologia Medica, II ${ }^{a}$ Facolta di Medicina, Università di Napoli, Italy, and Duke University Medical Center, Durham, North Carolina, USA

SUMMARY We describe a technique for interruption/modification of atrioventricular conduction 은 using a direct current shock delivered from a defibrillator to the atrioventricular junctional tissue by means of a conventional electrode wire. The method was used in three patients with refractory $\subseteq$ supraventricular tachycardia. After the procedure two patients received an atrioventricular sequen- $\vec{\Phi}$ tial pacemaker.

The first patient had a cardiomyopathy and intermittent Wolff-Parkinson-White syndrome result- $-\stackrel{\infty}{\infty}$ ing from a septal accessory atrioventricular pathway associated with a two year history of recurrent ${ }^{\circ}$ reciprocating tachycardia and occasional episodes of atrial flutter/fibrillation. Delivery of a direct $\stackrel{\text { s }}{3}$ current shock resulted in first degree atrioventricular block resulting from conduction delay proxi- $\frac{\bar{O}}{0}$ mal to the recorded His bundle deflection. Atrial pacing at a cycle length of $580 \mathrm{~ms}$ resulted in $2: 1 \stackrel{\circ}{\%}$ block and ventricular pacing showed no retrograde conduction. The patient has done well without $\stackrel{\perp}{\circ}$ pacemaker implantation and remains free from reciprocating tachycardia. During atrial flutter $/ \overrightarrow{0}$ fibrillation, the ventricular rate ranged from 62 to 75 beats/minute. These results are consistent with 3 modification of the atrioventricular node-His bundle, and complete ablation of conduction over the septal accessory pathway in both the anterograde and retrograde direction.

The second patient had coronary artery disease with a history of recurrent episodes of atrial $\overrightarrow{\mathbb{C}}$ fibrillation for two years. Delivery of a direct current shock resulted in persistent complete atrioven- $\frac{\mathscr{N}}{7}$ tricular block and neither anterograde nor retrograde conduction was present during atrial and $\frac{0}{3}$ ventricular pacing, respectively. During a sustained episode of atrial flutter, complete atrioventricu- - . lar block with a ventricular rate between 40 and 48 beats/minute was present. A permanent $\stackrel{\circ}{\ni}$ pacemaker was implanted.

The third patient had a 14 year history of incessant junctional reciprocating tachycardia. After the shock, sinus rhythm appeared with first degree block and anterograde conduction was evident foro the first time over a posterior septal pathway which exhibited decremental behaviour.

The follow-up is 15 months in the first two patients and 11 months in the third. We conclude that the technique used, which does not require open heart surgery, can provide effective treatment in $\tilde{D}^{\circ}$ patients with this disabling supraventricular arrhythmia.

Despite the availability of numerous antiarrhythmic drugs, supraventricular arrhythmias resistant to pharmacological treatment are not rare. Some of these

This study was supported in part by a grant from Consiglio Nazionale delle Ricerche, Rome, Italy.

Accepted for publication 8 February 1983 can be managed by pacemaker techniques. ${ }^{1-3}$ Nevertheless, when arrhythmias become disabling and unresponsive to the above mentioned approaches, surgical division of atrioventricular conduction witho implantation of a pacemaker should be considered. $\frac{4}{\mathrm{Q}}$ This approach, however, requires open heart surgery greatly limiting its use. 
Recently, a technique for closed chest interruption/modification of atrioventricular conduction was described. 56 In this communication we describe our experience with this technique in three illustrative cases.

\section{Subjects and methods}

The methods have been previously described. ${ }^{6}$ A 6 French bipolar electrode wire is passed transvenously with its tip positioned in the right ventricle and connected to a demand pacemaker. A second wire, a 6 French tripolar electrode wire (United States Catheter and Instrument Corporation), is advanced via the femoral vein to the region of the His bundle. Three unipolar electrograms are recorded simultaneously. The His bundle catheter is connected by means of a switching device to the cathodal output of a standard cardioversion (PhysioControl Lifepak 6) device. A back paddle, placed adjacent to the left scapula, is connected to the anodal output of the defibrillator. The switching device permits rapid shifting from recording to discharging. Thus, an attempt is made to record a His bundle electrogram which shows atrial and His bundle deflections of large amplitude. This is most commonly achieved on the distal unipolar electrode. The patient is then sedated with intravenous diazepam and preparations are made to discharge the defibrillator. A synchronised direct current shock is delivered through the appropriate electrode on the His bundle catheter. Demand ventricular pacing is instituted. The region of the His bundle is periodi- cally scanned with the pacemaker off in an attempt to record the electrical activity of the His bundle or the right bundle branch. To confirm the persistence of complete block, atropine ( $2 \mathrm{mg}$ IV) and isoprenaline ( $3 \mu \mathrm{g} / \mathrm{min}$ IV) are administered during atrial pacing.

After the procedure, demand ventricular stimulation is maintained at 70 beats $/ \mathrm{min}$ with continuous electrocardiographic monitoring. Serum cardiac isoenzymes of lactic dehydrogenase and creatine kinase are determined for $\mathbf{4 8}$ hours after the shock. After 24 to 72 hours, His bundle recordings and atrial pacing are repeated. An atrioventricular sequential pacemaker is implanted if persistent block is observed for at least 72 hours.

\section{CASE 1}

The first patient was a 61 year old man with cardiomegaly and intermittent pre-excitation syndrome caused by a posterior septal accessory pathway. He had a two year history of recurrent reciprocating tachycardia. Sporadic episodes of atrial flutter/ fibrillation had been documented as well. During an episode of atrial fibrillation, a pseudoventricular tachycardia resulting from maximal pre-excitation was documented. Amiodarone prevented a life threatening ventricular response during induced atrial fibrillation, but it failed to prevent the arrhythmia (Fig. 1). The ventricular rate during arrhythmic episodes averaged 150 to 160 beats/min and further pharmacological regimens with common antiarrhythmic drugs did not yield useful results. An automatic radiofrequency device ${ }^{7}$ also failed to provide

A : before

B: after Amiodarone

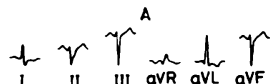

$$
\begin{aligned}
& l_{v 1} \bigwedge_{v 2} \downarrow_{v 3} \downarrow_{v 4} \underset{v 5}{\downarrow} \underset{v 6}{-1}
\end{aligned}
$$

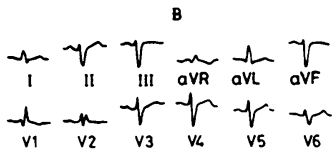

Induced atrial flutter-fibrillation

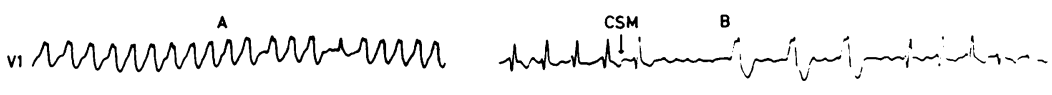

$$
\begin{aligned}
& \begin{array}{ll}
\text { Minimal R-R } & 240 \\
\text { Ventricular rate } & 160
\end{array} \\
& 96
\end{aligned}
$$

Fig. 1 Case 1. Amiodarone effects (2000 mg weekly for four weeks) on the pre-excitation pattern during both sinus rhythm and induced atrial flutter/fibrillation. After treatment pre-excitation signs disappear. Ventricular response during atrial tachyarrhythmia shows maximal pre-excitation in all beats but one, with a heart rate of $160 \mathrm{bpm}$; minimal $R R$ interval of subsequent pre-excited beats of $240 \mathrm{~ms}$. During amiodarone treatment no pre-excited beat is present, heart rate being 96 bpm with a right bundle-branch block pattern. Carotid sinus massage induces an increase in atrioventricular node refractoriness which results in a transient slowing of ventricular response and in the appearance of three pre-excited beats, probably caused by the shift of anterograde conduction from the nodal-His to the accessory pathway.

Alternatively this event might be explained in terms of complete atrioventricular block followed by three escape beats. 

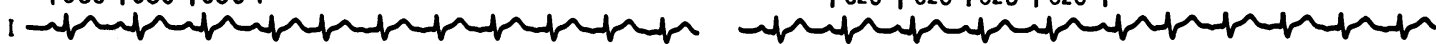

III
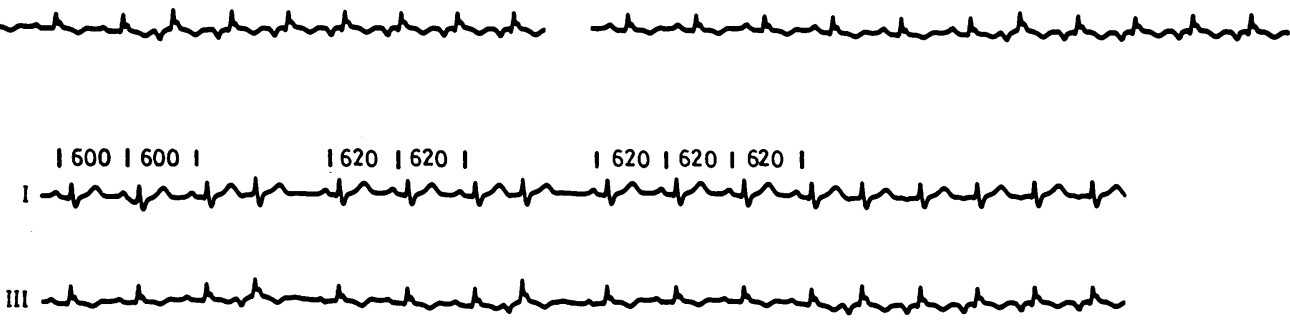

Fig. 2 Case 3. Reciprocating tachycardia showing PR shorter than RP interval. Neither critical PP interval nor critical atrioventricular delay is evident at tachycardia initiation.

control of recriprocating tachycardia.

\section{CASE 2}

The second patient was a 60 year old woman with ischaemic heart disease and a history of recurrent episodes of atrial flutter/fibrillation; long term treatment with antiarrhythmic drugs was ineffective and the patient had undergone cardioversion on one occasion. Cardiomegaly and signs of congestive heart failure were present.

\section{CASE 3}

The third patient was a 36 year old woman who had suffered for 14 years from incessant iunctional reciprocating tachycardia, unresponsive to pharmacological treatment. During tachycardia, heart rate ranged from 130 to 180 beats/min and the surface electrocardiograms showed a retrograde $P$ wave, with a $P R$ interval shorter than RP interval (Fig. 2). Sinus rhythm showed a normal PR interval and no evidence of a delta wave. Neither critical cycle length nor critical atrioventricular conduction was found to be necessary for tachycardia initiation. During elec- trophysiological investigation, anterograde and $\stackrel{\circ}{3}$ retrograde curves could not be analysed because of the $\vec{c}$ presence of incessant tachycardia. Endocardial map- $\frac{\overline{5}}{\overline{5}}$ ping of the right atrium and coronary sinus showed ${ }^{\widehat{D}}$ (Fig. 3) that the earliest retrograde atrial electrogram $\vec{\oplus}$ was recorded in the region of the coronary sinus $\omega$ orifice. Premature ventricular stimulation at coupling intervals ranging from 390 to $325 \mathrm{~ms}$ interrupted tachycardia without resulting in retrograde atrial activation. Ventricular premature beats delivered at a® time when the His bundle was refractory could also interrupt tachycardia.

\section{Results}

Two shocks were necessary in all patients to obtain useful results. A first shock of 200 joules resulted in $\vec{\varnothing}$ transient block of conduction in the atrioventricular node-His bundle in all cases, with restoration of con- $\overline{-}$ duction after 30 minutes, three hours, and 20 days, respectively. Electrode wire positioning, however, was impaired in each of these cases by the presence of $₹$ atrial flutter/fibrillation, atrial flutter, and reciprocat- -9

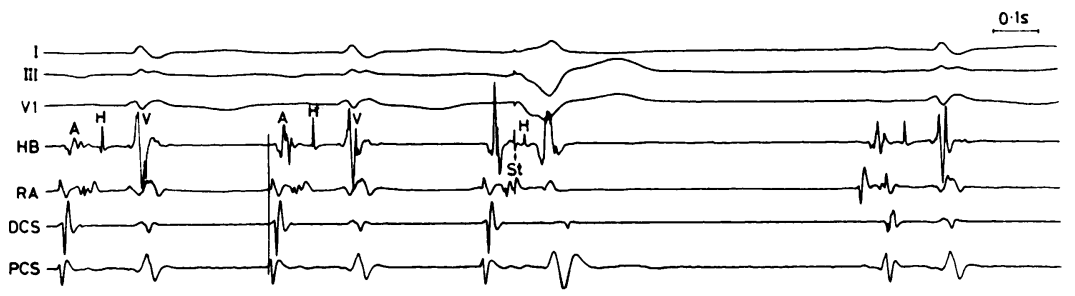

Fig. 3 Case 3. Endocardial mapping of right atrium and coronary sinus during reciprocating tachycardia (first two beats), and its interruption by premature ventricular stimulation. The earliest activation occurs on the proximal coronary sinus (PCS), indicating that the accessory pathway has its atrial end near the coronary sinus orifice. Premature ventricular stimulation interrupts the tachycardia circuit in the retrograde limb, since no retrograde atrial activation occurs. The fourth beat is a sinus rhythm beat. 
ing tachycardia, respectively, at the time of the initial attempt.

In case 1 , a second shock of 280 joules delivered during sinus rhythm produced complete atrioven-
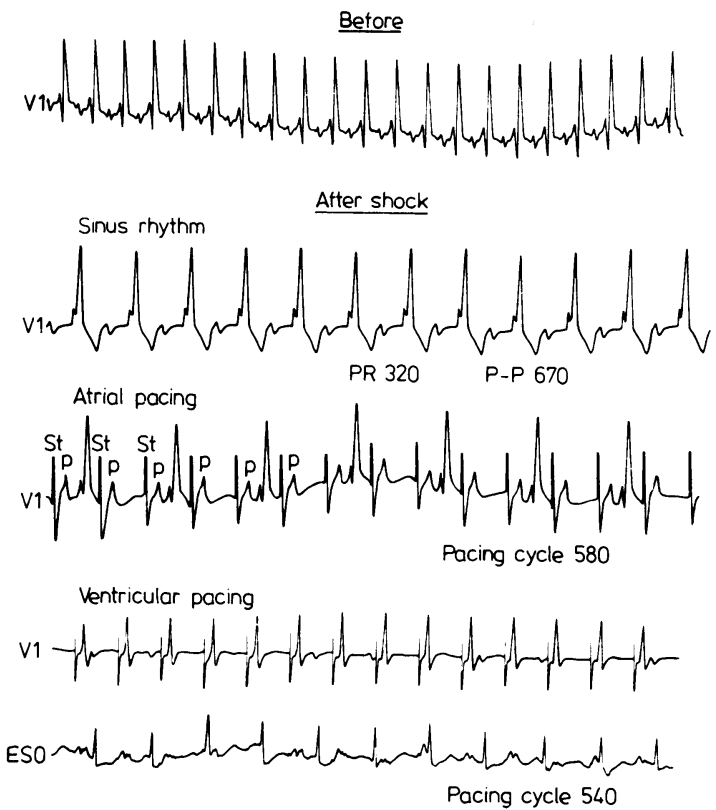

Fig. 4 Case 1. Surface electrocardiogram recorded during reciprocating tachycardia before the shock and during both sinus rhythm and atrial and ventricular pacing after the shock. See text for explanation. ESO, transoesophageal atrial electrogram.

Numbers indicate ms. tricular block with junctional escape rhythm; three days later a second degree block of the type I variety appeared, followed by $1: 1$ conduction with delay proximal to the recorded His deflection (AH interval $240 \mathrm{~ms}$ ). Atrial pacing at a cycle length of $580 \mathrm{~ms}$ induced 2:1 atrioventricular block and ventricular pacing showed the absence of retrograde conduction (Fig. 4). After the shock (Fig. 5), the ventricular rate during atrial flutter/fibrillation ranged from 62 to 75 beats/min in contrast with the fast rate observed before the shock. These results have been confirmed during repetitive tests of anterograde and retrograde conduction during the follow-up period.

In case 2 , the second shock ( 280 joules) delivered during sinus rhythm resulted in persistent complete atrioventricular block with an escape rhythm of 50 beats/min; right bundle-branch block was present as a result of the first shock. Atrial and ventricular pacing after atropine documented the absence of anterograde and retrograde conduction. The ventricular response during a sustained episode of atrial flutter ranged from 40 to 48 beats $/ \mathrm{min}$. These results were reproducible in the follow-up period.

In case 3, the first shock of 200 joules had resulted in disappearance of reciprocating tachycardia for three weeks. During that time, the surface electrocardiogram showed sinus rhythm with first degree atrioventricular block (PR interval 0.36 s). The QRS morphology was anomalous. There was extreme left axis deviation and the precordial forces showed a transition complex in V1 with positive complexes noted in V2 to V6. An electrophysiological study performed 20 days after the first shock (Fig. 6) showed the absence of anterograde conduction over the His

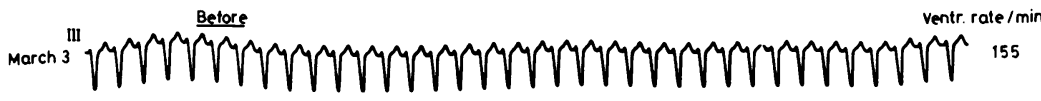

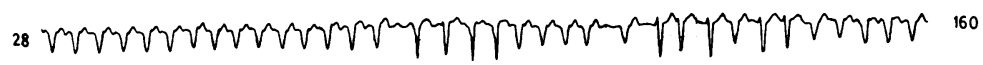

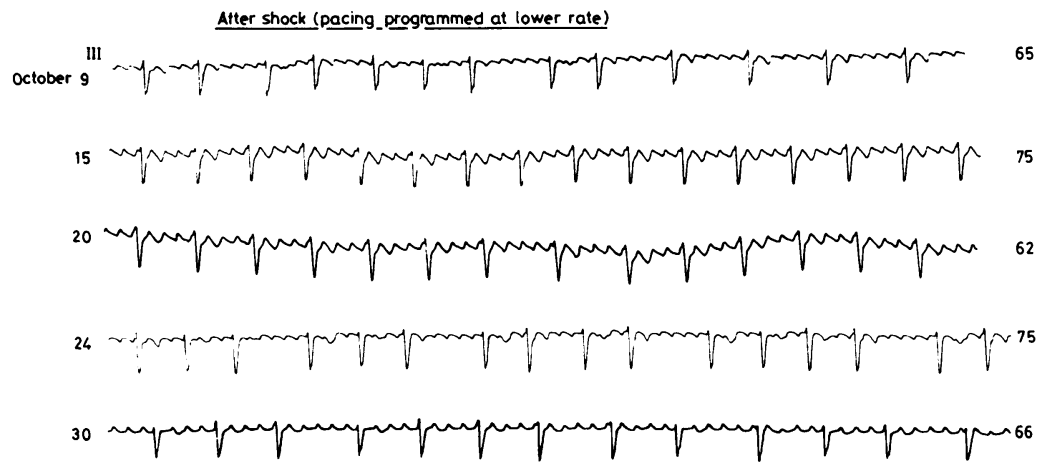

Fig. 5 Case 1. Electrocardiographic tracing recorded before the shock and during the follow-up. See text for explanation. 


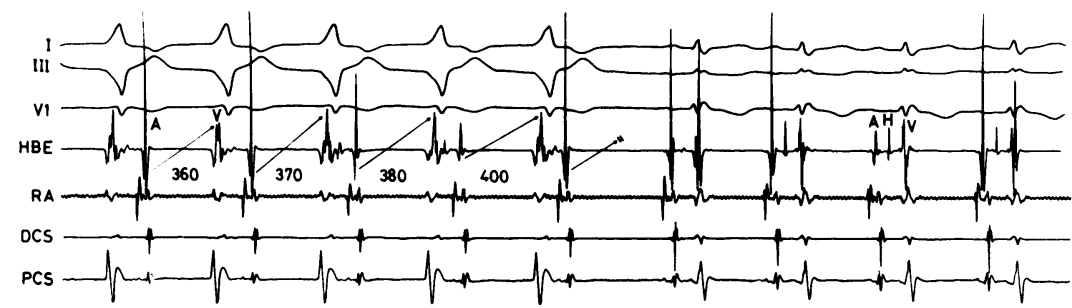

Fig. 6 Case 3. Surface electrocardiogram $(I, I I I, V 1)$ and endocavity recordings obtained 20 days after the first shock. Sinus rhythm with Wenckebach type block and no anterograde Hisian potential are present (first five complexes). At the end of the Wenckebach phenomenon a change in the atrioventricular conduction pattern appears, with normal intervals and right bundle-branch block. See text for further details. HBE, His bundle electrogram; RA, right atrium electrogram; DCS, distal coronary sinus; $P C S$, proximal coronary sinus. Numbers indicate ms.

bundle when anomalous QRS complexes were evident. During the study, however, a spontaneous atrioventricular Wenckebach phenomenon was observed. This was followed by a sudden change in the atrioventricular conduction pattern, characterised by evidence of His bundle activation with normal atrioventricular conduction intervals. The same transition to normal conduction could be elicited by atrial pacing at a cycle length of $560 \mathrm{~ms}$. Subsequently, tachycardia recurred. A second shock of 280 joules delivered during sinus rhythm resulted in first degree atrioventricular block (PR 0.36 s). The AH interval measured $90 \mathrm{~ms}$. All conducted atrial impulses were now associated with anomalous $Q R S$ responses which were not preceded by His bundle deflections.

A repeat electrophysiological study was performed 30 days later. Spontaneous atrioventricular Wenckebach was again observed. Administration of atropine as well as atrial pacing at a cycle length of $580 \mathrm{~ms}$ resulted in 2:1 atrioventricular conduction. Once again, all conducted atrial impulses manifested an anomalous QRS complex. There was no evidence of conduction over the atrioventricular node-His bundle. Ventricular pacing at a cycle length of $480 \mathrm{~ms}$ resulted in earliest retrograde atrial activation in the region of the orifice of the coronary sinus. A retrograde Wenckebach phenomenon followed by $2: 1$ ventriculoatrial conduction appeared at a cycle length of $380 \mathrm{~ms}$. Since the second shock, the patient has remained free of tachycardia. The above observations were felt to be consistent with anterograde (and retrograde) conduction over a septal accessory pathway exhibiting decremental behaviour.

\section{Discussion}

The various techniques previously used in attempts to interrupt atrioventricular conduction have yielded inconsistent results, with a variable incidence of

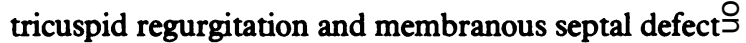
noted. Such methods include ligation of tissue near ${ }^{-}$ the atrioventricular node, electrocautery of the nodal-His region, mechanical crushing, surgical dis-ब section, septal infarction, and formalin injection in $\overrightarrow{0}$ the region of the His bundle. ${ }^{4} 6$ Recently a new technique of ablating atrioventricular conduction using a cryoprobe has been introduced. ${ }^{89}$

In this communication, the possibility of obtaining closed chest interruption/modification of atrioventricular conduction without septal or tricuspid valve damage while preserving a subsidiary escape rhythm $\stackrel{2}{\rightarrow}$ is shown. Furthermore, as documented by the studyô of cardiac enzyme release, no relevant myocardial damage results from the use of this method. ${ }^{6}$

As to the energy level, a 200 joules discharge should suffice in the presence of a correct position of the electrode. Nevertheless, in our patients a second shock of 280 joules was required. Correct cathetero positioning during the first attempt was probably?. affected by the presence of atrial tachyarrhythmia ino all three patients such that a large atrial electrogram $₹$ was not observed at that time.

In the first patient, clinical results were considered optimal for the following reasons: (a) the appearance․ㅡ. of 2:1 atrioventricular block during atrial pacing at an cycle length of $580 \mathrm{~ms}$ as well as the absence of retrograde conduction during ventricular pacing shows 0 that a reciprocating tachycardia cannot occur (Fig. 4). Indeed, since the second shock this patient has been? free from such episodes of tachyarrhythmia; (b)o furthermore, the absence of anomalous anterogradē conduction as well as the absence of any retrograde? conduction suggests that the accessory pathwayo responsible for pre-excitation was ablated. The damage provoked by the shock is responsible for a high? degree of atrioventricular block during atrial tachyar $\frac{\overline{0}}{2}$ rhythmias resulting in only a moderate ventricularo response. Though the method described in this paper 
usually requires pacemaker implantation, the results obtained in this first patient suggest the theoretical possibility of modifying atrioventricular conduction without interrupting it. ${ }^{10}$ Proper positioning of the catheter as well as appropriate energy levels might permit limited damage of atrioventricular conduction.

The results obtained in the third patient are probably the result of interruption of the His bundle with anterograde conduction now occurring over a slow conducting accessory pathway. Before the shock, this accessory pathway was evidenced only by retrograde conduction. Observations made after the two shocks in this patient show that an "unusual" form of intranodal re-entry"1 was not responsible for tachycardia in this case and suggest instead that the tachycardia circuit incorporated a slowly conducting accessory ventriculoatrial pathway. ${ }^{12-15}$ The presence of intact anterograde conduction over this accessory pathway at present avoids pacemaker dependence in this patient.

Although the application of the method was confined in this communication to the atrioventricular junction, the potential clearly exists in the future for use of this technique as a means for treatment of tachycardias arising in the atrium, other portions of the atrioventricular junction, or the ventricle.

\section{References}

1 Critelli G, Grassi G, Perticone F, Adinolf L, Maione S, Condorelli M. Stimulation avec scansion automatique à radiofréquence dans le traitement à long terme des arythmies hypercinétiques. Arch Mal Coeur 1978; 71: 1279-82.

2 Critelli G, Grassi G, Chiariello M, Perticone F, Adinolfi L, Condorelli M. Automatic "scanning" by radiofrequency in the long-term electrical treatment of arrhythmias. PACE 1979; 2: 289-96.

3 Critelli G, Adinolfi L, Perticone F, Condorelli M. Programmed electrical stimulation and amiodarone therapy for the control of persistent junctional tachycardia. Cardiovasc Dis 1981; 8: 232-7.
4 Sealy WC, Gallagher JJ, Kasell J. His bundle interruption for control of inappropriate ventricular responses to atrial arrhythmias. Ann Thorac Surg 1981; 32: 429-38.

5 Gonzalez R, Scheinman MM, Margaretten W, Rubinstein M. Closed-chest electrode-catheter technique for His bundle ablation in dogs. Am f Physiol 1981; 241: H283-7.

6 Gallagher JJ, Svenson RH, Kasell J, et al. Catheter technique for closed-chest ablation of the atrioventricular conduction system. $N$ Engl f Med 1982; 306: 194-200.

7 Critelli G, Grassi G, Adinolfi L, Perticone F, Condorelli M. Arrhythmia control by cardiac stimulation. Eur $\mathcal{f}$ Cardiol 1981; 12: 297-307.

8 Harrison L, Gallagher JJ, Kasell J, et al. Cryosurgical ablation of the A-V node-His bundle. A new method for producing A-V block. Circulation 1977; 55: 463-70.

9 Klein GJ, Sealy WC, Pritchett ELC, et al. Cryosurgical ablation of the atrioventricular node-His bundle: longterm follow-up and properties of the junctional pacemaker. Circulation 1980; 61: 8-15.

10 Pritchett ELC, Anderson RW, Benditt DG, et al. Reentry within the atrioventricular node: surgical cure with preservation of atrioventricular conduction. Circulation 1979; 60: 440-6.

11 Wu D, Denes P, Amat-y-Leon F, Wyndham CRC, Dhingra R, Rosen KM. An unusual variety of atrioventricular nodal re-entry due to retrograde dual atrioventricular nodal pathways. Circulation 1977; 56: 50-9.

12 Coumel P, Cabrol C, Fabiato A, Gourgon R, Slama R. Tachycardie permanente par rythme réciproque. Arch Mal Coeur 1967; 60: 1830-64.

13 Gallagher JJ, Sealy WC. The permanent form of junctional reciprocating tachycardia: further elucidation of the underlying mechanism. Eur $\mathcal{F}$ Cardiol 1978; 8: 41330.

14 Brugada P, Vanagt EJ, Bar FWHM, Wellens HJJ. Incessant reciprocating atrioventricular tachycardia. Factors playing a role in the mechanism of the arrhythmia. PACE 1980; 3: 670-7.

15 Epstein ML, Stone FM, Benditt DG. Incessant atrial tachycardia in childhood: association with ratedependent conduction in an accessory atrioventricular pathway. Am F Cardiol 1979; 44: 498-504.

Requests for reprints to Dr John J Gallagher, Box 3816, Duke University Medical Center, Durham, North Carolina 27710, USA. 